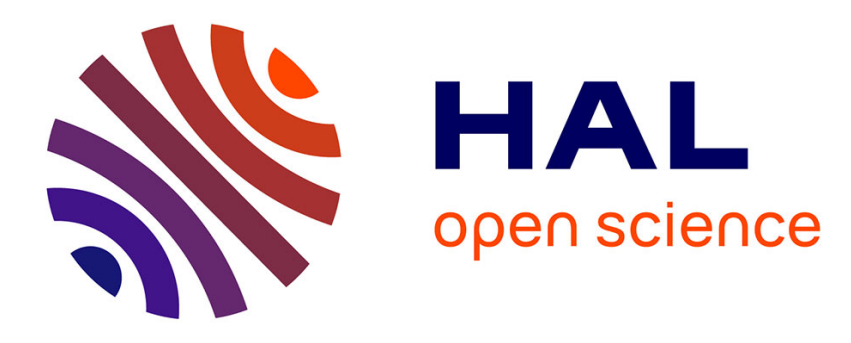

\title{
Quand le droit compte comme texte
}

Pierre Brunet

\section{To cite this version:}

Pierre Brunet. Quand le droit compte comme texte. Revue Interdisciplinaire d'Etudes Juridiques, 2013, 70 (1), pp.54-59. halshs-00839725

\section{HAL Id: halshs-00839725 \\ https://shs.hal.science/halshs-00839725}

Submitted on 29 Jun 2013

HAL is a multi-disciplinary open access archive for the deposit and dissemination of scientific research documents, whether they are published or not. The documents may come from teaching and research institutions in France or abroad, or from public or private research centers.
L'archive ouverte pluridisciplinaire HAL, est destinée au dépôt et à la diffusion de documents scientifiques de niveau recherche, publiés ou non, émanant des établissements d'enseignement et de recherche français ou étrangers, des laboratoires publics ou privés. 


\title{
QUAND LE DROIT COMPTE COMME TEXTE
}

Revue Interdisciplinaire d'Études Juridiques,

2013/1, numéro spécial Le droit en contexte, p. 54-59.

\author{
Pierre Brunet \\ Professeur à l'Université Paris Ouest, Directeur de l'UMR CNRS 7074 Centre de \\ théorie et analyse du droit, Membre de l'Institut Universitaire de France
}

1. Le droit est-il source, affluent, alluvion ou aboutissement des changements sociaux?

N'étant pas sociologue du droit, je cours le risque de ne parvenir à répondre aux questions posées autrement qu'en égrenant quelques banalités sur la circularité de la relation entre le droit et le changement social.

Il serait à mon sens assez présomptueux de tenter de savoir qui, du droit ou des changements sociaux, détermine l'autre. Il y a à cela une raison logique : c'est que les changements sociaux sont à la fois le résultat du droit, un usage de ce dernier et toujours un « jeu » avec lui (pour reprendre une métaphore qui eut son heure de gloire). Et dans le même temps, le droit joue aussi avec les mœurs et est déterminé par eux, que ce soit pour y résister ou pour s'y conformer.

Mais ce sont là des généralités creuses : tout le droit n'est pas déterminé par les changements sociaux et ce ne sont pas tous les changements sociaux qui sont en permanence déterminés par le droit. Il y a sans cesse un effet de rétroaction de l'un sur les autres et les fins que l'on assigne à une norme juridique ne sont souvent atteintes que par hasard (lorsqu'elles le sont). Les sociologues du droit ont écrit et continuent d'écrire sur ce sujet des pages bien meilleures que je ne pourrais le faire. Weber lui-même se donnait justement comme objet d'étudier la façon dont les acteurs utilisent les règles, se servent d'elles dans une situation donnée et sont déterminés par elles ${ }^{1}$. La difficulté demeure, toujours, de parvenir à reconstruire la chaîne causale.

La difficulté vient aussi de ce que les termes «droit», « société », « changement social » sont très indéterminés. Bien évidemment, on serait tenté de se faciliter la tâche en prenant le «droit » dans son sens impérativiste et un peu naïf, de règles émanant d'un souverain et qui s'imposent aux sujets. Mais plus personne ne croit - y a-t-on jamais cru ? - à une telle conception et nous savons bien que rien n'est plus délicat que d'identifier le «droit positif». Les juristes d'aujourd'hui sont,

\footnotetext{
${ }^{1}$ Max Weber, Économie et Société, trad. fçse Jacques Chavy and Eric de Dampierre, Vol. 2. L'organisation et les puissances de la société dans leur rapport avec l'économie, Paris, Agora, p. 43-44 : "certaines régularités de fait du comportement ("coutume") peuvent être à l'origine de règles de comportement ("convention", "droit"). Mais l'inverse est vrai aussi. Non seulement les normes (conventionnelles ou) juridiques produisent ou contribuent à produire les régularités mêmes auxquelles tendent leurs injonctions, mais elles en produisent d'autres encore ».
} 
plus encore qu'hier, des photographes contrariés qui peinent à régler la vitesse de leurs appareils, non seulement parce que le matériau qu'ils doivent saisir s'est enrichi et ne cesse de proliférer mais aussi parce que les photographies se multiplient.

2. Qu'est-ce, pour moi, qu'une approche du droit « en contexte »? Quels en sont les enjeux, les atouts et les limites?

On pourrait de prime abord penser qu'une telle question appelle une réponse simple et un peu péremptoire : sauf à céder à un formalisme aveugle, il n'y a pas d'approche du droit qui puisse ne pas être « en contexte ». Le problème est qu'il n'y a pas " une » approche du droit en contexte mais plusieurs (et donc plusieurs contextes).

Ce n'est pas le lieu ici de faire la généalogie de l'expression « droit en contexte ». À ma connaissance, elle a été proposée comme un synonyme de « réalisme » par William Twining dans un article de $1967^{2}$. Depuis, dans la littérature «scientifique» contemporaine, l'approche dite «en contexte» désigne les nombreuses formes qu'a pu prendre la contestation du « positivisme juridique » et plus précisément encore du "formalisme ». Cette dernière expression est alors utilisée avec une forte connotation péjorative pour dénoncer une conception qui réduit le droit à un système logique, clos, complet et cohérent au sein duquel les juristes et les juges pourraient, à l'aide de méthodes scientifiques, ou du moins rigoureuses, déduire des décisions et jugements corrects, exacts, à partir de quelques principes et concepts fondamentaux, où le droit est vu à la fois comme une science et comme un ensemble de normes susceptibles d'être l'objet d'une connaissance distincte de ce système. L'amusant est que, si on y regarde bien, le premier à avoir proposé une étude du droit « en contexte » est peut-être Bentham et son analyse métajuridique du mot «droits » à travers laquelle il parvient à montrer que le terme n'a aucune référence et que sa signification ne peut être saisie autrement que dans le contexte de son utilisation. Or, par bien des aspects, la théorie du droit de Bentham a tout d'un formalisme. On pourrait donc le dire autrement : l'approche «en contexte » rejette une étude du droit tel qu'il est dans les livres (« law in books») au profit d'une approche du droit tel qu'il se fait ( law in action »). L'étude du «droit en contexte» est donc à l'opposé de cette idée que le droit serait un système conceptuel fermé sur lui-même. On connaît la fameuse phrase de Holmes contre Langdell (« Law is not logic but experience ») qui a inspiré bien du monde. Se réclamant justement de Holmes, les juristes réalistes américains n'ont eu de cesse de souligner l'importance de nombreux

\footnotetext{
${ }^{2}$ William Twining, « Two Works of Karl Llewellyn (Part 1) », The Modern Law Review, Vol. 30, $\mathrm{N}^{\circ} 5,1967$, p. 514-30, ici p. 529 : «A working theory of law which guides detailed work and which in its turn is tested and developed by regular application to concrete problems. In the United States some theories of this kind were crudely characterised as "realism". In the United Kingdom "realism" has acquired such ridiculous associations that an alternative term is needed. Perhaps "a contextual approach" is sufficiently clear and sufficiently open-ended ». Elle était également apparue un peu plus tôt chez lui, dans un texte non publié : R. Stevens et W. Twining, «Law in Context : A Tentative Rationale » (Unpublished paper presented to Weidenfeld and Nicholson), Avril, 1966.
} 
facteurs non juridiques ou extra juridiques dans la production du droit et la très faible part que prend la logique dans le raisonnement des juges. Le prolongement de leurs thèses, ou le point d'aboutissement de certaines d'entre elles, fut la consécration d'une approche sociologique du droit qui s'est ensuite réclamée de diverses filiations.

Il peut aujourd'hui paraître quelque peu contradictoire de se réclamer du réalisme sans adopter la sociologie du droit qui semble aller de pair. Pourtant, nombre de théoriciens du droit aujourd'hui se disent réalistes sans abandonner le point de vue du juriste au profit de celui du sociologue.

D'une certaine façon, ils ont été précédé par d'autres, qui se réclamaient aussi du réalisme et luttaient alors contre le positivisme autant que le droit naturel. On pense ici aux juristes réalistes scandinaves qui opposèrent à Kelsen son propre formalisme en insistant sur la contradiction qu'il y avait à vouloir faire une «science du droit» qui ne prenne jamais en compte les faits humains et ne connaissent que des entités non factuelles relevant du monde du Sollen et de la pure « validité ». En dépit de leurs divergences et différence d'approche, le point commun aux réalismes américains et scandinaves est très certainement le goût pour les faits et donc le contexte dans lequel se déploie la pratique juridique. On se souvient de l'attention avec laquelle Llewellyn scrutait la façon dont les juges d'appel examinaient les faits et tenaient compte des pratiques dominantes dans tel ou tel milieu. À sa façon, il soulignait l'importance de s'attacher à ce que les juges font et aux justifications juridiques qu'ils utilisent dans leurs décisions, la fameuse « legal doctrine ». De ce point de vue, il faut rappeler qu'il regrettait la façon dont certains critiques ou commentateurs réduisaient ses propres thèses à quelques formules à l'emporte-pièce dont il était familier (dont, par exemple celle selon laquelle « what officials do about disputes is, to my mind, the law itself» ${ }^{3}$ ).

Le problème que pose l'étude "en contexte» est alors non de savoir où commence le contexte mais bien de déterminer où il s'arrête. Autrement dit, doiton y inclure la composition du petit déjeuner des juges (ou de toute autorité conduite à prendre une décision) et l'état de leurs relations conjugales (ou extra conjugales), etc?

Le contexte auquel s'attachent les théoriciens réalistes s'étend à - s'entend de tout ce que font les juristes avec le matériau juridique - leur pratique des textes et des interprétations de ces textes : un contexte juridique donc. Or, cette pratique consiste en actes de langage, en production de concepts, en rationalisations qui engagent les juristes pour l'avenir et tissent peu à peu un ensemble contraignant. Dans une conception réaliste rigoureuse, le droit positif n'est pas perçu comme un ensemble de règles obligatoires, qui s'imposeraient en quelque sorte par nature à tout organe d'application du droit (tout « law maker»). Parler de textes ici ne doit d'ailleurs pas prêter à confusion : il ne s'agit nullement de réduire le droit à une forme de raisonnement purement déductif où le « texte » serait unique, doté de la

\footnotetext{
${ }^{3}$ The Bramble Bush, «Foreword» (2 éd. 1951), reproduit ds l'édition dirigée par S. Sheppard, Oxford UP, 2008.
} 
force inouïe de l'évidence qui le ferait s'imposer par lui-même et d'un sens vrai qu'il parviendrait à révéler à ceux qui le lisent ou l'entendent. Il ne s'agit pas davantage de privilégier un système de droit écrit au détriment d'un système de droit «commun ». On parle de textes car, qu'il soit écrit ou non, on n'est encore jamais parvenu à saisir le droit autrement que par le langage. Par ailleurs, toute autorité qui exerce du pouvoir dans un système constitutionnel (non autocratique) ne l'exerce jamais seul et doit tenir compte du pouvoir - et même de la compétence juridique - de nombreuses autres autorités.

Le contexte juridique est donc aussi institutionnel que matériel et les juristes concernés sont autant ceux qui disposent d'un pouvoir de décision que ceux qui ne disposent que d'un pouvoir de suggestion. À cet égard, il faut insister sur le rôle de la doctrine - dogmatique - juridique qui ne peut pas, ne doit pas être laissée de côté. Les interprétations et systématisations qu'elle produit sont précieuses en ce qu'elles fournissent un grand nombre d'informations sur la façon dont pensent les juristes et dont ils se représentent le droit positif. Une approche réaliste du droit, qui entend rendre compte du droit « en contexte » ne peut donc guère se passer d'un examen de cette doctrine. Mais encore faut-il ajuster le regard et soumettre son discours à une analyse sinon logique du moins critique ${ }^{4}$. Par là, il ne s'agit nullement de prendre la position du maître d'école mais, plus simplement, de ne pas se laisser abuser par la recherche, souvent immodérée en doctrine, de cohérence et de complétude de l'ordre juridique, les deux mamelles de la sécurité du droit, cette drogue des juristes. Pour le dire encore autrement, la doctrine fournit bien souvent un grand éventail d'énoncés que Hart aurait appelé « internes » et qui sont, en réalité, des normes - des énoncés normatifs ${ }^{5}$. Certes, leur efficacité est faible si on se place du point de vue des justiciables ou des organes d'application du droit. Mais ils contribuent à tracer un cadre contraignant prima facie - des interprétations attendues, raisonnables, possibles voire souhaitables.

Et c'est encore une analyse en contexte que se propose de mener une théorie dites des «contraintes juridiques» qui s'attache à reconstruire ex post facto, les fameuses «contraintes » proprement juridiques qui ont conduit telle autorité à choisir, parmi toutes celles qui se présentaient à elle, une signification plutôt qu'une autre ${ }^{6}$. Cette théorie se réclame elle aussi d'une étude du droit en contexte et réduit ce contexte au droit positif. Ce dernier est alors entendu comme un ensemble d'arguments disponibles et mobilisables au gré des finalités que poursuivent ces mêmes organes. Ainsi, en tant que tel, ou, pour le dire autrement, tant qu'ils ne sont pas mis en relation avec une fin spécifique, ces arguments demeurent dépourvus de signification. Mais c'est précisément dans le contexte

\footnotetext{
${ }^{4}$ Cf. G. Tarello, Diritto, enunciati, usi : studi di teoria e metateoria del diritto, Bologna, Il Mulino 1974.

G. Tarello, L'interpretazione della legge, Milano, Giuffré, 1980 ; R. Guastini, Lezioni sul linguaggio giuridico, Torino, Giappichelli, 1985.

${ }^{6}$ M. Troper, V. Champeil-Desplats, C. Grzegorczyk (dir.), Théorie des contraintes juridiques, LGDJ, Paris, 2005.
} 
d'une décision et de la construction d'une justification que ces arguments se voient attribuer une signification propre. Or, si le contexte ici s'entend dans un sens juridique, il s'étend aux systèmes de justification que mobilisent tous les organes d'application susceptibles d'entrer en conflit les uns avec les autres.

Les limites sont donc assez aisément perceptibles : étudier le droit en contexte, dans cette perspective là, c'est ne pas sortir d'une conception du droit réduite sinon à des textes du moins à des argumentations juridiques ou, pour le dire autrement, à un langage voire à une forme d'exercice du pouvoir à l'aide du langage (plus encore que de la force physique). Peut-être l'expression américaine de «legal doctrine» peut-elle ici nous aider car elle recouvre justement, tel qu'elle est employée dans le discours juridique américain, tout cet arsenal juridique disponible qui ne demande qu'à être utilisé. Mais cette limite n'empêche cependant pas une compréhension du phénomène que l'on étudie et semble même assez pertinente eu égard à son objet. Elle peut par ailleurs être heureusement complétée par d'autres travaux s'attachant eux-mêmes à un contexte plus large. 